Document downloaded from:

http://hdl.handle.net/10251/66535

This paper must be cited as:

Doménech Antich, EM.; Martorell Alsina, SS. (2016). Definition and usage of food safety margins for verifying compliance of food safety objectives. Food Control. 59:669-674. doi:10.1016/j.foodcont.2015.05.042.

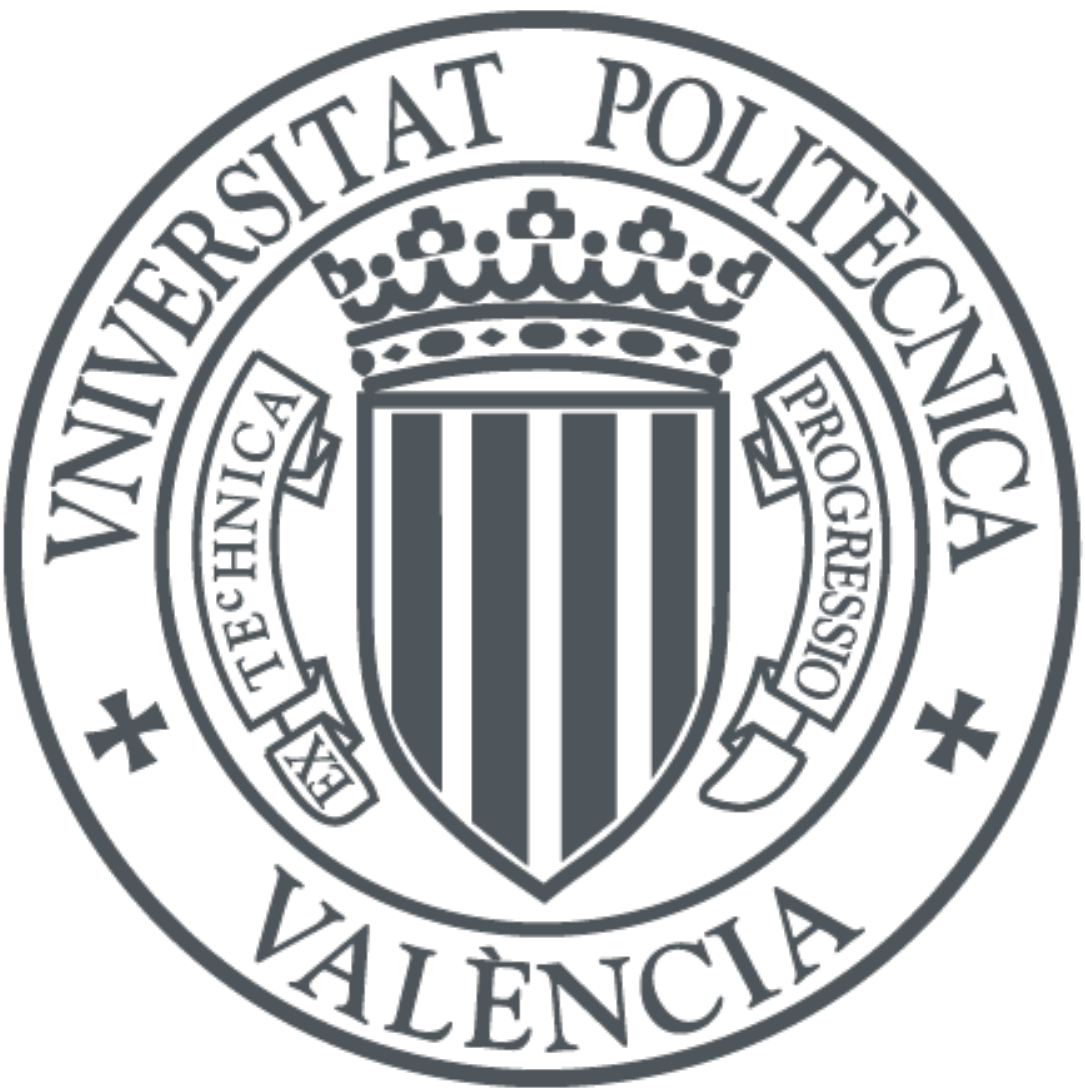

The final publication is available at

https://dx.doi.org/10.1016/j.foodcont.2015.05.042

Copyright Elsevier

Additional Information 


\title{
Definition and usage of Food Safety Margins for verifying compliance of Food Safety Objectives
}

\author{
Eva Domenech $^{1, *}$, Sebastian Martorell ${ }^{2}$ \\ ${ }^{1}$ Institute of Food Engineering for Development (IUIAD), Department of Food \\ Technology (DTA) \\ ${ }^{2}$ Department of Chemical and Nuclear Engineering \\ UNIVERSITAT POLITÈCNICA DE VALÈNCIA
}

\begin{abstract}
Over the last decade, risk analysis has been gradually introduced as a tool to make decisions about food safety policies. In this framework, the ALOP (Appropriate Level of Protection) concept, which can be seen as a statement of the degree of public protection that is to be achieved within a country, was introduced. In addition, FSO (Food Safety Objectives) was introduced to provide a link between the ALOP and target points/goals in the supply chain. Historically, ALOP and FSO decisions have been based on the ALARA (As Low As reasonably Achievable) approach. Since an ALARA approach is based on the status of current technology, it is likely that the ALOP is achievable, provided a substantial portion of the industry complies with technological requirements or adopt "best practices" that will achieve the FSO. Food managers must control and government agencies must enforce and monitor fulfilment of FSO. Once FSO has been established and adopted by the food industry, a major concern is to evaluate the results of the implementation of FSO in order to verify compliance. This paper introduces the concept of food safety margin (FSM) and its formulation based on classical and probabilistic approaches, which are intended to be used as a tool to measure the degree of compliance with FSO. For a better understanding of how food safety margins perform, FSM are estimated for L. monocytogenes in three different products, (i.e. semi-soft cheese, heat treated meat and cold smoked salmon). The results obtained, adopting both classical and probabilistic approaches, are discussed.
\end{abstract}

Keywords: FSO, Exposure assessment, risk management

\section{Introduction}

Globalization of food markets as well as the fact that hazards, e.g. microbial growth, can appear at any stage of the food chain has increased regulators' concern about the new challenges to manage food related risks to human health to guarantee food safety.

Over last decade, risk analysis has been gradually introduced as a tool to make decisions on food safety management policies. Following the Commission's Green Paper on food law (COM, 1997), and subsequent consultations, a new legal framework was proposed. This covers the whole of the food chain, including animal feed production, establishing a high level of consumer health protection and clearly attributing primary responsibility for safe food production to the industry, producers and suppliers. The White Paper on food safety proposed risk analysis as the baseline of food safety policy (CCE, 2000). The European Union (EU), through Regulation (EC) No 178/2002 laid down the general principles and requirements of food law, emphasizing 
that in order to achieve the general objectives of a high level of protection of human health, food law shall be based on risk analysis. In 2007 the Codex Alimentarius Commission published the principles of risk analysis for food safety to be applied by governments. The standard was intended to provide guidance to national governments for risk assessment, risk management and risk communication with regard to food related risks to human health (CAC, 2007a). More recently, the European Food Safety Agency (EFSA) highlighted risk analysis as the starting point for setting priorities and allocating resources effectively based on risk (EFSA, 2012).

Risk assessment provides a systematic means for assessing, in a qualitative or quantitative way, the probability of occurrence and the severity of known or potentially adverse health effects in a given population based on hazard identification, hazard characterization, exposure assessment and risk characterization. The results obtained through risk assessment are the foundations of good safety/risk management policies.

Risk management is defined for the purposes of the Codex Alimentarius Commission as the process, distinct from risk assessment, of weighing policy alternatives, in consultation with all interested parties, considering risk assessment and other factors relevant for the health protection of consumers and for the promotion of fair trade practices, and, if needed, selecting appropriate prevention and control options (CAC, 2007b). Thus, the reduction of potential hazards associated with foods typically involves the application of preventive and control measures in the food chain, from primary production to consumption. Therefore, one important aspect of risk management involves verification of the effectiveness of these measures and their capability to control the hazard. These goals have traditionally been managed through the establishment of Microbiological Criteria, Process Criteria, and Product Criteria (CAC, 2007b). However, these traditional safety criteria have not generally been linked directly to a specific level of public health protection (CAC, 2007b; Manfreda \& De Cesare, 2014).

To advance in risk management, new risk management tools emerged where food safety issues moved from a hazardbased approach to a risk-based approach (CAC, 2007b). Consequently, safety goals had to be developed taking into account the levels of illness associated with a pathogen/food combination, and the need for a continuous improvement in public health, while acknowledging that zero risk cannot be attained (Walls, 2006). In this framework, the ALOP (Appropriate Level of Protection) concept was introduced in the World Trade Organization Agreement on the Application of Sanitary and Phytosanitary Measures (ICMSF, 2002). The ALOP is viewed as a statement of the degree of public protection that must be achieved within a country as a consequence of the presence of a hazard in a food. One difficulty when implementing the ALOP concept is that the ALOP cannot be used directly by the food industry or government regulatory agencies to set a target for food safety systems (Doménech et al., 2012).

The International Commission on Microbiological Specifications for Foods (ICMSF, 2002) proposed the establishment of FSO (Food Safety Objectives) to provide a link between the ALOP and target points in the supply chain. The FSO, which is defined as the maximum frequency and/or concentration of a microbial hazard in a food considered tolerable for consumer protection at the time of consumption, conveys the ALOP criteria into targets/goals that can be controlled by food producers and monitored by 
government agencies, (CAC, 2004). FSO is a risk management decision that is based on both scientific and societal attributes, whose role is to establish the level of residual risk in a food safety system that is tolerable. FSO can be used by Government regulatory agencies to communicate public health goals to the industry and other stakeholders in a form that can provide a measurable target for a hazard (Walls \& Buchanan, 2005). In order to adapt the principles of FSO implementation at earlier stages in the food chain, they are linked to Performance Objectives (PO). A PO is the maximum level (frequency and/or concentration) of a hazard in a food at a specified point in the food chain that should not be exceeded in order to achieve an FSO (CAC, 2004). A major concern is to set up the FSO and the corresponding $\mathrm{PO}$ values that must attain the FSO established in order to guarantee consumer's health. Walls \& Buchanan (2005) emphasised that setting an FSO involves determination of the maximum level of exposure to comply with public health goals, which must include consideration of the need to take into account the variability in food safety management performance and uncertainty in the knowledge of risk. The role of the FSO is to establish the level of residual risk in a food safety system that is tolerable. The $\mathrm{PO}$ at specific points of the food production chain must be set up by industries linked to such FSO.

As the $\mathrm{FAO} / \mathrm{WHO}$ remarked in (2002), historically, ALOP and FSO decisions have been based on the ALARA (As Low As reasonably Achievable) approach. Since an ALARA approach is based on the status of current technology, it is likely that the ALOP is achievable, provided a substantial portion of the industry complies with technological requirements or adopt "best practices" that will achieve FSO. However, a potential limitation of this approach is that unrealistic public health goals could be specified that are not achievable by industry within a realistic time frame.

Food managers must control, and government agencies must enforce and monitor fulfilment of FSO. Therefore, once the FSO has been established and adopted by the food industry, a major concern is to verify the results of the implementation of FSO after the adoption of technological requirements or "best practices" by food industries.

This paper introduces the concept of food safety margin (FSM) that is intended to be used as a tool to measure the degree of compliance of FSO goals in a quantitative way, which could be used by government regulatory agencies, the food industry and other stakeholders. Two metrics, i.e. the classical and the probabilistic approach, are provided. For a better understanding of how food safety margins perform, FSM are estimated for L. monocytogenes in three different products, (semi-soft cheese, heat treated meat and cold smoked salmon). Finally, results obtained adopting both classical and probabilistic approaches are discussed.

\section{Dose-Response. A link between ALOP and FSO}

Dose-response analysis involves the study of the characterization of the relationship between dose, infectivity and the likelihood and severity of spectrum of adverse health effects associated with the hazard, $\mathrm{H}$, in an exposed population (Walls, 2006). Thus, the probability that a person shows an adverse effect after consuming a product with a given toxic/microbiological load, $\mathrm{D}(\mathrm{H})$; is used to determine the FSO necessary to achieve the ALOP depending also on the expected population being exposed to this risk.

The probability density function (pdf) of the dose-response curve, $\mathrm{D}(\mathrm{H})$, is affected not only by the level of a hazard 
$\mathrm{H}(\log (\mathrm{CFU} / \mathrm{g}))$ but also by numerous parameters: the virulence of the pathogen, the age and immune status of the person, the food matrix (fat level, acidity) and the treatments received by the product. Therefore, establishing a value for the FSO of one particular food is complex and it may be one of the main reasons why numeric safety goals have not been regulated yet for FSO. Thus, numerical values of $\mathrm{MC}$ are often adopted instead of such numerical safety goals to represent FSO.

\section{Exposure Assessment and FSO}

In this context, exposure assessment is defined as the quantitative evaluation of the likely human intake of biological, chemical and physical hazards via food (CAC, 2013). When characterizing and quantifying exposure to microbial pathogens of one individual, data on the frequency of contamination (prevalence) and the numbers of microorganisms (concentration) in a specific food are needed. The amount of food consumed for estimating public exposure is also needed (Walls, 2006). Figure 1 shows the relationship between the concept of Exposure-assessment, E(H), and FSO defined for a hazard, $\mathrm{H}(\log (\mathrm{CFU} / \mathrm{g}))$. In Figure 1, two situations of Exposure assessment, $\mathrm{E}_{1}(\mathrm{H})$ and $\mathrm{E}_{2}(\mathrm{H})$, with different proportion of unsatisfactory results are represented. Thus, $\mathrm{E}_{2}(\mathrm{H})$ shows the highest proportion of unsatisfactory results, i.e. major probability of the exposure to the hazard exceeding the FSO, so-called exceedance probability in Figure 1, which may be associated with worse risk management practices than the first one. The relationship between the $\mathrm{E}(\mathrm{H})$ and the FSO is used in the coming sections.

\section{Validation of compliance of FSO. Variability and uncertainty}

Validation of food management involves the collection and evaluation of documented evidence in order to determine whether control measures are capable of achieving their specified purpose in terms of hazard control and deliver a specified performance objective (Swanson \& Anderson, 2000; Keener, 2006). In particular, validation involves measuring performance of a desired food safety criterion with respect to a required level of hazard control (CAC 2008). This is how the validation process demonstrates that the selected control measures are actually capable, on a consistent basis, of achieving the intended level of hazard control.

Once the FSO has been established, comparison between FSO and exposure assessment can be used to determine whether a specific food, considering the whole food chain, achieves its safety goal. Then, this comparison can be used to validate the whole food process (Whiting \& Buchanan, 2008). Compliance of FSO for a specific food can be verified in a quantitative way using the equation (1) proposed by the ICMSF (ICMSF, 2002).

$H=H o-\sum R+\sum I \leq F S O$

Where $\mathrm{H}_{0}$ is the initial level of the hazard units, $\sum R$ is the total (cumulative) reduction of the hazard, $\sum I$ is the total (cumulative) increase of the hazard and FSO is the food safety objective. All terms are expressed in $\log _{10}$ units (Walls, 2006).

Eqn. (1) establishes that the initial contamination, minus reductions through inactivation steps, plus potential recontamination and possible growth during storage should be the pathogen level at the time of consumption, i.e. $\mathrm{H}$, which should be below a specific level in every serving, i.e. FSO.

As one can envisage from the several contributions in Eqn. (1), FSO is a useful concept for translating the obtained results of control measures, including those which are applied at the level of 
consumer, into public health outcomes (FAO/WHO, 2006). For this reason, FSO is an important component of a riskbased system of food safety. By setting an FSO, competent authorities articulate a risk-based limit that should be achieved operationally within the food chain, while providing flexibility for different productions, manufacturing, distribution, marketing, and preparation approaches.

Eqn. (1) can be reformulated in its simplest form as proposed in Eqn. (2) to be used for verifying compliance of FSO of a specific food. Thus, since $\mathrm{H}=\mathrm{Ho}-$ $\sum R+\sum I$, then:

FSO $-H \geq 0$

The use of deterministic models is often a common practice to estimate the level of a hazard, H. However, generally, a deterministic model generates a conservative assessment of the risk (FAO/WHO, 2006; Whiting, 2011). This way, Eqn (2) would result in a conservative estimation of food safety.

In the framework of risk analysis it is more appropriate to use a probabilistic model to represent the level of a hazard. Exposure assessment, $\mathrm{E}(\mathrm{H})$, is an example of the use of such a probabilistic model to represent the presence of a given level of a hazard, $\mathrm{H}$. Thus, a probabilistic or stochastic model of $\mathrm{E}(\mathrm{H})$ permits a more realistic formulation of the condition for verifying compliance of FSO, then, Eqn. (2) can be particularized as follows (see graphical representation in Figure 1):

$F S O-E(H) \geq 0$

However, the use of such a realistic model in Eqn, (3), also known as a "best estimate (BE)" model, provides, generally, less conservative results for such a comparison of $\mathrm{H}$ against FSO, which can be affected largely by uncertainty. Uncertainties can be categorized as either "random" or "epistemic" (Doménech et al., 2010). Random uncertainty reflects our inability to predict random observable events. It represents a true heterogeneity of the population that is a consequence of both biological and physical system and irreducible by further measurements, so that, it is often referred as "variability". Epistemic uncertainty represents our lack of knowledge. It is also called "state-of-knowledge uncertainty" or just "uncertainty" (Doménech et al., 2010). There are many types of epistemic uncertainty including: 1) process uncertainty (relates to the process conditions), 2) model uncertainty (each model has a different approximation of the same real-world system), 3) scenario uncertainty (linked to the portion of risk that is not explicitly included in the analysis), 4) parameter uncertainty, or even 5) uncertainty in variability (FAO/WHO, 2008, Doménech et al., 2010; Basset et al., 2012).

Therefore, a better way to validate compliance of FSO based on Eqn. (3) is adopting a BEPU (Best Estimate Plus Uncertainty) approach, which uses realistic models and accounts for uncertainties in an integrated manner (Pagani, 2004). Thus, Exposure Assessment must provide an estimate with associated uncertainty of the occurrence and level of the pathogen in a specified portion of a certain food at the time of consumption in a specified population. Separation of uncertainty and variability in predictive models used for Exposure assessment is considered to be of increasing importance in several fields of risk analysis, including the agro-food sector (Nauta, 2000).

\section{Definition of Food Safety Margin}

No universal definition exists of the term safety margin. Pagani (2004) introduced both probabilistic and classical definitions of safety margins. 
There, safety margins are used to deal with uncertainties related to the concept of functional failures. A functional failure is defined as the inability of a system to perform its mission due to deviations from its expected behaviour.

The concept of safety margin is used linked to real systems performance to deal with the uncertainties associated with safety of the design, operation and management of such systems and their impact on safe performance of such systems. Then, this concept has a direct connection with safety goals imposed on systems performance. Thus, if safety margin is positive, this means that the safety goal is met.

The concept of safety margin is adapted for the food industry in this paper. Thus, a Food Safety Margin (FSM) is set in relation to the FSO. This validates that not only is FSO met under the normal performance of the food chain but also that FSM deals with failures in the food chain to perform in a safe way due to deviations from the expected behaviour.

Adoption of the "probabilistic" approach to FSM can be expressed in general terms as the probability that the load (exposure assessment) exceeds the capacity (dose-response). This definition results from the comparison of $\mathrm{E}(\mathrm{H})$ against $\mathrm{D}(\mathrm{H})$.

A more practical FSM can be introduced to assess up to what level the food chain meets the imposed safety goals and how it is affected by uncertainties. This definition fits well to the realistic formulation of the condition for verifying compliance of FSO given by Eqn. (3), which makes use only of the relationship between $\mathrm{E}(\mathrm{H})$ and $\mathrm{FSO}$ in Figure 1. Again, adopting the "probabilistic" approach to FSM, it is defined as the probability that the exposure assessment (load) is less than the FSO (see Figure 1).

Alternatively, following the same practical application that makes use of
Eqn. (3) but adopting now the "classical" approach to FSM, it can be defined as the normalized difference between the calculated value of exposure $\mathrm{E}(\mathrm{H})$ and the FSO considered as a threshold.

\section{Formulation of Food Safety Margin}

In this section, we present the basis of the quantification of the FSM based on the use of Eqn. (3) and adopting both "classical" and "probabilistic" approaches to FSM definition. To quantify the FSM, it is necessary to adopt the FSO established and to obtain the exposure assessment, $\mathrm{E}(\mathrm{H})$, to a given load of a Hazard, $\mathrm{H}$, at time of consumption. This exposure can be derived as the result of a simulation through the whole food chain or of a sampling at retail, addressing variability and uncertainty.

The FSM can be formulated in its classical form (c_FSM), Eqn. (4), adopting a normalized distance between $\mathrm{E}(\mathrm{H})$ and FSO based on the use of Eqn. (3):

$C_{-} F S M=\frac{F S O-E(H)}{F S O}$

Since $\mathrm{E}(\mathrm{H})$ represents a continuous distribution function, Eqn. (4) provides another distribution function for c_FSM. Therefore, single statistics of the c_FSM can be obtained, for example the mean value, percentile $5 \%$ or percentile $95 \%$.

Alternatively, the FSM can be formulated in its probabilistic form (p_FSM), Eqn (5), which is derived from Eqn. (3) as follows:

$p_{-} F S M=\operatorname{Pr}\{F S O-E(H) \geq 0\}=$ $\int_{0}^{F S O} E(H) d H=1-\int_{F S O}^{\infty} E(H) d H=1-$ EP

where parameter EP (Exceedance Probability) represents the fraction of 
food exceeding the FSO (see Figure 1). Note Eqn (5) provides a single value.

\section{Examples of Food Safety Margin calculation}

Safety margins were studied in three different products: Semi soft cheese, heat treated meat and cold smoked fish. All of them have different origins and process conditions. The microorganism considered in this case of application was Listeria monocytogenes. Contamination data for this microorganism in these products at retail were based on the analysis of the baseline survey on the prevalence of L. monocytogenes in certain ready-to-eat foods in the EU, 2010-2011 published by the European Food Safety Authority (EFSA, 2013). Table 1 shows the distributions obtained for each product that were got by fitting the published data with the software @ Risk 5.7 (Palisade Newfield). Moreover, only random uncertainty (i.e. variability) of field data has been considered in this example of application.

An official numerical safety goal value for FSO has not been published. However, the FAO/WHO proposed that the level of $L$. monocytogenes in readyto-eat foods must not exceed $100 \mathrm{cfu} / \mathrm{g}$ when they are consumed (FAO/WHO, 2002). This value has been adopted for FSO in this paper for ready-to-eat food throughout its shelf life.

Tables 2 and 3 show the results of the quantification of both FSM, i.e. c_FSM and p_FSM for these products, which were obtained with the application of Eqns. (4) and (5) respectively.

\section{Discussion}

Food safety has been traditionally, and will continue to be, the responsibility of industry based on how well it is capable of performing, i.e., by implementing the ALARA "as low as reasonably achievable" criteria rather than based on establishing a degree of stringency (Walls, 2006). In addition, the increase of the international food trade has favoured the appearance of the concept of equivalence with the objective of providing the same level of protection in the different countries.

Risk analysis has been gradually introduced as a tool to support decisionmaking on food management policies aimed at improving food safety in this framework of globalization where food safety principles must follow ALARA criteria. In this context, implementation of ALOP, FSO and PO principles plays a key role.

There is a wide consensus on the fact that this implementation will not be a simple task. Thus, Walls and Buchanan in (2005) indicated that one of the considerations to set an FSO is to determine the maximum level of exposure plus an extra margin that should be added in order to take into account the variability and the uncertainty. With the same idea, the CAC in (2007b) highlighted the difficulty in translating the results of risk assessment into a set of simple limits, which could be communicated and implemented. In the same way, Whiting in (2011), concluded that exposure assessment links the sources of contamination with their evolution through the stages of the food chain to determine whether the process meets the FSO or PO and ultimately the acceptable level of risk to the consumer (ALOP).

Nowadays, one of the main challenges is to establish a value for the FSO since, only microbiological criteria for foodstuffs have been regulated (Regulation (EC) No 2073/2005). The ICMSF in (2005) indicated that governments should determine through discussions with relevant experts and stakeholders what FSO values should be feasible. In some cases, it may turn out that it is not possible to comply with a set FSO level in practice, and a government 
may decide to set a less stringent FSO or to provide a period during which processing procedures can be changed to meet the FSO.

However, no matter on what basis numerical safety goals of concern to FSO, and PO, will be finally established in the future, which would have to guarantee an acceptable ALOP, another major challenge is verification of FSO compliance by industry.

This paper introduce the concept of FSM and proposes two quantitative approaches, i.e. classical and probabilistic, as a tool to measure the degree of compliance of FSO based on the comparison of exposure assessment against FSO. The formulation of this new risk metric following the classical approach allows estimating the normalized distance between $\mathrm{E}(\mathrm{H})$ and FSO. Alternatively, the probabilistic approach allows estimating the probability that $\mathrm{E}(\mathrm{H})$ does not violate the corresponding safety limit FSO, or, complementarily, it allows estimating the exceedance probability.

Having a quantitative measure of safety margin allows us to assess whether the FSM is sufficient or not. The new risk metric proposed in this paper will allow also obtaining an estimation of the increase or reduction of a safety margin after any change in food chain conditions, i.e. comparing the safety margin before and after the change.

One advantage of adopting the probabilistic approach to FSM is to obtain the exceedance probability in a probabilistic risk assessment context, such as the one adopted for QMRA (quantitative microbiological risk assessment), which is based on the use of predictive modelling adopting probabilistic models, which typically employ unbounded distributions (e.g., log-normal distributions for microbial populations). However, the classical approach to FSM is more appropriate when safety concern, i.e. Exposure
Assessment, is far away from the safety limit, i.e. FSO. In such a case, EP would most probably be zero, so any change to food chain conditions could not be measured in terms of its impact on the probabilistic FSM. Anyhow, and at least in the nearest future, both lines of work, classical and probabilistic approaches to FSM, should be maintained in parallel.

The result of the examples of FSM calculations shows FSM is high for all three foods (see for example Table 3). The results are coherent with available filed data showing that high levels of contamination appears only in a minority of servings, which are responsible of foodborne illnesses representing the portion of risk implicitly accepted based on the ALOP proposed for these foods.

\section{References}

Bassett, J., Nauta, M., Lindqvist, R., \& Zwietering, M. (2012). Tools for microbiological risk assessment. Report Commissioned by the ILSI Europe Risk Analysis in Food Microbiology Task Force. January http://www.ilsi.org/Europe/Publications/MR A\%20Tools.pdf (Accessed December 2014).

CAC. (2004). Report of the twentieth session of the Codex committee on general principles. Codex Alimentarius Commission Paris, France, ALINORM 04/27/33A. file://ftp.fao.org/codex/alinorm04/al0433ae. pdf. (Accessed December 2014).

CAC. (2007a). Working principles for risk analysis for food safety for application by governments. Codex Alimentarius Commission $\quad \mathrm{CAC} / \mathrm{GL}$ 62-2007. http://www.codexalimentarius.org /standards/list-of-standards/es/?provide= standards\&orderField $=$ fullReference $\&$ sort $=$ asc $\&$ num $1=\mathrm{CAC} / \mathrm{GL}$ (Accessed December 2014).

CAC. (2007b). Principles and guidelines for the conduct of microbiological risk management. Codex Alimentarius Commission CAC/GL 63-2007. http://www.codexalimentarius.org /standards/list-of-standards/es/?provide $=$ standards\&orderField $=$ fullReference $\&$ sort $=$ asc $\&$ num $1=\mathrm{CAC} / \mathrm{GL}$ (Accessed December 2014).

CAC. (2008). Guidelines for the validation of food safety control measures. Codex Alimentarius Commission CAC/GL 69 - 
2008. http://www.codexalimentarius.org /standards/list-ofstandards/es/?provide=standards\&orderField $=$ fullReference $\&$ sort $=$ asc \&num $1=\mathrm{CAC} / \mathrm{GL}$ (Accessed December 2014)

CAC. (2013). Guidelines on the application of risk assessment for feed. Codex Alimentarius Commission $\quad$ CAC/GL 80-2013. http://www.codexalimentarius.org /standards/list-ofstandards/es/?provide=standards\&orderField $=$ fullReference $\&$ sort $=$ asc \&num $1=\mathrm{CAC} / \mathrm{GL}$ (Accessed December 2014).

CCE. (2000) White Paper on Food Safety. Commission of the European Communities. Bruselas 12-1-2000. COM (1999) 719 final. http://ec.europa.eu/dgs/health_consumer/libr ary/pub/pub06_es.pdf COM (1999) 719 final.

Doménech, E., Martorell, S., \& Escriche, I. (2010). Exposure Assessment based on a combination of event and fault tree analyses and predictive modelling. Food Control, 21, 1338-1348.

Doménech, E., Amorós, J.A., Martorell, S., \& Escriche, I. (2012). Safety assessment of smoked fish related to Listeria monocytogenes prevalence using risk management metrics. Food Control, 25, 233238.

EFSA. (2012). Scientific opinion on the development of a risk ranking framework on biological hazards. EFSA Journal 10: 2724. http://www.efsa.europa.eu/ de/search/doc/2724.pdf.

(Accessed December 2014).

EFSA. (2013). Analysis of the baseline survey on the prevalence of Listeria monocytogenes in certain ready-to-eat foods in the EU, 20102011 Part A: Listeria monocytogenes prevalence estimates. EFSA Journal 2013;11(6):324.

http://www.efsa.europa.eu/en/efsajournal/pu b/3241.htm (Accessed December 2014).

FAO/WHO. (2002). Principles and guidelines for incorporating microbiological risk assessment in the development of food safety standards, guidelines and related texts. Report of a Joint FAO/WHO Consultation. ftp://ftp.fao.org/docrep/ fao/006/y4302e/y4302e00.pdf.

FAO/WHO. (2006). The use of microbiological risk assessment outputs to develop practical risk management strategies: metrics to improve food safety.

FAO/WHO. (2008). Exposure assessment of microbiological hazards in foods: Guidelines. Microbiological Risk Assessment Series No. 7. WHO Press, Geneva. http://www.fao.org/docrep/010/a0251e/a025 1e00.htm (Accessed December 2014)
ICMSF. (2002). Microorganisms in Foods 7 microbiological testing in food safety management. International Commission on Microbiological Specifications for Foods New York, NY: Klewer Academic, pp. 290291.

ICMSF. (2005). A simplified guide to understanding and using Food Safety Objectives and Performance Objectives. The International Commission on Microbiological Specifications for Foods. http://www.icmsf.org/pdf/FSO\%20Ojectives / GuiaSimplificadoEnglish.pdf (Accessed December 2014)

Keener, L. (2006). Hurdling new technology challenges: investing in process validation of novel technologies. Food Safety Magazine (February/March issue). http://www.foodsafetymagazine.com/article. asp? $\mathrm{id}=490 \&$ sub $=$ sub 1 .

Manfreda, G., \& De Cesare, A. (2014). The challenge of defining risk-based metrics to improve food safety: Inputs from the BASELINE project. International Journal of Food Microbiology, 184, 2-7.

Nauta, M.J. (2000). Separation of uncertainty and variability in quantitative microbial risk assessment models. International Journal of Food Microbiology, 57, 9-18.

Pagani, L. (2004). On the Quantification of Safety Margins. PhD Thesis. Massachusetts Institute of Technology, September 2004. http://dspace.mit.edu/bitstream/handle/1721. 1/33642/64394636.pdf (Accessed December 2014).

Regulation (EC) No 2073/2005 of 15 November 2005 on microbiological criteria for foodstuffs Official Journal of the European Union L 338/1-26. https://www.fsai.ie/uploadedFiles/Reg2073 2005(1).pdf (Accessed December 2014).

Regulation (EC) No 178/2002 of the European Parliament and of the Council of 28 January 2002 laying down the general principles and requirements of food law, establishing the European Food Safety Authority and laying down procedures in matters of food safety. $\mathrm{L}$ 31, 1-24. http://eurlex.europa.eu/LexUriServ/

LexUriServ.do?uri=OJ:L:2002:031:0001:00 24:EN:PDF. (Accessed December 2014).

Swanson, K.M.J., \& Anderson, J.E. (2000). Industry perspectives on the use of microbial data for hazard analysis and critical control point validation and verification. Journal of Food Protection, 63, 815-818.

Walls, I. \& Buchanan, R.L. (2005). Use of food safety objectives as a tool for reducing foodborne listeriosis. Food Control, 16, 795799. 
Walls, I. 2006. Role of quantitative risk assessment and food safety objectives in managing Listeria monocytogenes on readyto-eat meats. Meat Science 74, 66-75.

Whiting, R.C., \& Buchanan, R.L. (2008). Using risk assessment principles in an emerging paradigm for controlling the microbial safety of foods. In D. W. Schaffner (Ed.), Microbial risk analysis of foods (pp. 29-50). Washington, D.C: ASM Press.

Whiting, R.C. 2011. What risk assessments can tell us about setting criteria. Food Control, $22,1525-1528$

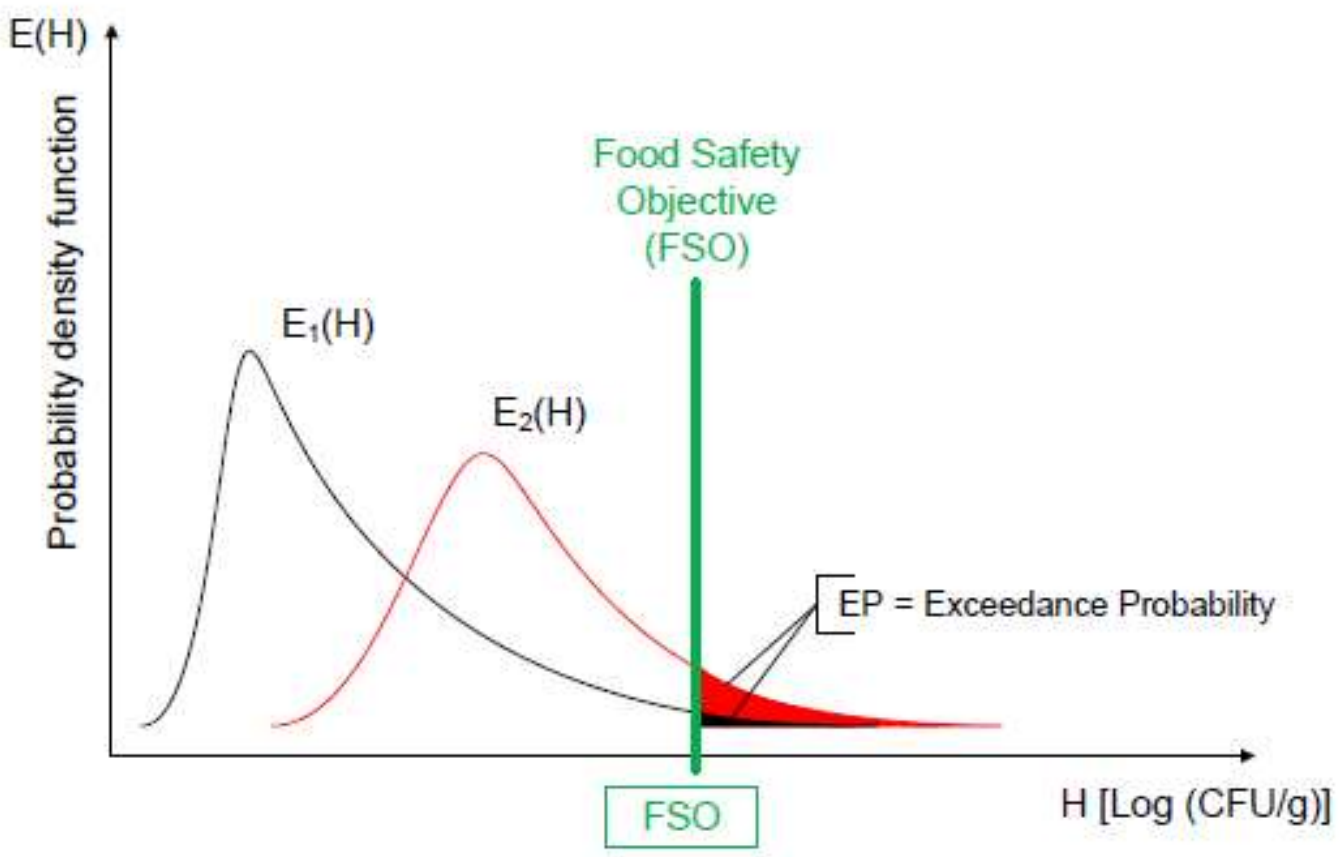

Fig. 1. Graphical relationship between Exposure assessment (E) and FSO in relation to a hazard $(H)$ for two feasible cases, $E_{1}(H)$ and $E_{2}(H)$.

Table 1 Probabilistic density function of the load (CFU/g) at retail for the different products (EFSA, 2013)

\begin{tabular}{ll}
\hline Product & Distribution \\
\hline Semi-soft cheese & Logistic $(-6.1781 ; 11.417 ; \operatorname{RiskTruncate}(0 ;))$ \\
Heat treated meat & Logistic $(-13.313 ; 15.703 ; \operatorname{RiskTruncate}(0 ;))$ \\
Cold smoked fish & Extvalue $(-52.033 ; 23.782 ; \operatorname{RiskTruncate}(0 ;))$
\end{tabular}


Table 2. Results for classical FSM, i.e. c_FSM

\begin{tabular}{lccc}
\hline Product & Mean & Quartile 5\% & Percentile 95\% \\
\hline Semi-soft cheese & 0,858 & 0,608 & 0,991 \\
\hline Heat treated meat & 0,813 & 0,476 & 0,989 \\
\hline Cold smoked fish & 0,755 & 0,275 & 0,987 \\
\hline
\end{tabular}

Table 3. Results for probabilistic FSM, i.e.p_FSM

\begin{tabular}{lll}
\hline Product & EP (\%) & p_FSM (\%) \\
\hline Semi-soft cheese & 0.03 & 99.97 \\
\hline Heat treated meat & 0.24 & 99.76 \\
\hline Cold smoked fish & 1.58 & 98.42
\end{tabular}

